\title{
ENSEMBLE METHODS FOR ECG-BASED HEARTBEAT CLASSIFICATION
}

\author{
Rui Duan, Sabah Mohammed and Jinan Fiaidhi \\ Department of Computer Science, Lakehead University, Thunder Bay, Ontario \\ $P 7 B$ 5E1, Canada \\ \{rduan, sabah.mohammed, jfiaidhi\}@lakeheadu.ca
}

\begin{abstract}
Ensemble method is a meta-algorithm to build strong classifiers based on a set of weak classifiers. This work explores some ensemble classifiers on UCI Arrhythmia Dataset to classify the heartbeat records. Each record corresponds to a person, and is the features extracted from the person's raw ECG data over a period. Two popular ensemble classifiers XGBoost and RandomForest are used, and the classic LogisticRegression is tried as a comparison. These classifiers are applied on all the 279 features in the dataset, and predict the heartbeat categories for the records. XGBoost and RandomForest perform better than even well-tuned LogisticRegression. We build VotingClassifier based on the ensemble voting metaalgorithm and the above three built-in classifiers, and it outperforms even welltuned XGBoost and RandomForest. The best prediction accuracy $76 \%$ is achieved by the VotingClassifier in this multiclass classification problem. This result is comparable to many other findings that uses similar or different classifiers.
\end{abstract}

Keywords - ECG classification, XGBoost, Random Forest, Logistic Regression, Ensemble Method, Scikit-learn

\section{INTRODUCTION}

An electrocardiogram (ECG) measures the electric activity of the heart and has been widely used for detecting heart diseases due to its simplicity and non-invasive nature. The last decades have seen a lot of paper researching on ECG-based heartbeat classification using supervised learning algorithms. The classification can identify the normal heartbeat and the abnormal ones. The abnormal heartbeat is called cardiac dysrhythmia (or arrhythmia) which occurs when the electrical activity of the heart is irregular. Effects of arrhythmia range from discomfort to cardiac arrest. Although most arrhythmias are harmless, arrhythmia is still responsible for about 500,000 deaths in the US, annually. Early detection and treatment of arrhythmia can reduce the number of deaths by $25 \%$ [6]. A full automatic system for arrhythmia classification can help the healthcare providers analyze ECG. Such kind of classification system can be divided in four steps, as follows: (1) ECG signal preprocessing; (2) heartbeat segmentation; (3) feature extraction; and (4) learning/classification [7]. The survey [7] introduces the state-of-the-art methods in the literature for all these four steps. In this work, step (4) is focused. We explore and compare three supervised learning classifiers by doing the multiclass classification on UCI Arrhythmia Dataset, and use an ensemble method to improve the performance. The three classifiers are: gradient boosting, forests of randomized trees, and logistic regression. Gradient boosting and forests of randomized trees are two kinds of ensemble methods. 


\section{CRITICAL REVIEW}

The ECG signal is obtained from a lead. A lead is a connector to an electrode. An electrode is a conductive pad in contact with the body that makes an electrical circuit with the electrocardiograph. Since leads can share the same electrode, a standard 12-lead ECG happens to need only 10 electrodes. The signal of a lead is the electrical potential difference between the two electrodes. In the literature, the researchers usually use the 10 electrodes configuration, and use lead II for diagnosing heart diseases. The raw cardiac analog signal passes a high-pass band filter, an amplification, a low-pass band filter, then the ADC converts the signal to ECG digital signal which is the input to the anomaly detector program.

\subsection{PROBLEM DOMAIN}

A full automatic system for arrhythmia classification from signals acquired by an ECG device can help answer the following 2 sets of questions which are grouped by two different criteria, and these problems are studied for decades.

Group 1: anomaly detection and multiclass arrhythmia classification.

Question 1a: Is it an anomaly?

Question 1b: What type of arrhythmia is it?

Anomaly detection answers question 1a, multiclass arrhythmia classification answers question 1b. Anomaly detection is in effect a two-class arrhythmia classification, although in the literature, they are usually solved in different approaches. Anomaly detection is usually solved by time series raw data analysis, and arrhythmia classification is usually solved by machine learning approaches.

Group 2: per-heartbeat classification and per-person classification.

Question 2a: What kind is this heartbeat?

Question 2b: In general, what kind of heartbeat is this person?

To answer question $2 \mathrm{a}$, a segmented heartbeat sample is analyzed and classified. To answer question $2 \mathrm{~b}$, a sample record for a person's heartbeat in a period is analyzed and classified. UCI Arrhythmia Dataset consists of records for a few persons with each record for one person, and each record is a set of features extracted from the raw ECG time series data. Hence UCI Arrhythmia Dataset is for question 2b. While the classic MIT-BIH Arrhythmia Dataset is good for question 2a.

\subsection{RESEARCH PROBLEM DEFINITION}

Question $2 b$ is answered in this work. Step (4) learning/classification is focused. The input is the UCI Arrhythmia Dataset, and the output is the arrhythmia types for the records in the test set which is part of the UCI dataset. Exploring the dataset helps clarifying the research problem.

2.3.1. UCI ARRHYTHMIA DATASET: The records of UCI Arrhythmia Dataset capture the statistical characters of a given person. Using this dataset, our classification aims at identifying what kind of arrhythmia a person has, it is not per-heartbeat oriented but per-person oriented.

The UCI Arrhythmia Dataset consists of 452 records with 279 attributes per record. Each record is assigned to 1 of 16 classes: a class label of 1 indicates normal ECG patterns while a class label between 2 to 16 indicates "abnormal ECG patterns" or arrhythmia of varying types. The class distribution is shown in Fig. 1. Each record in the dataset is derived from the ECG raw data obtained from a distinct person by the 12 ECG leads (DI, DII, DIII, AVR, AVL, AVF, V1, V2, V3, V4, V5, and V6). Each record is a 
set of statistical features whose values have been measured using the IBM-Mt. Sinai Hospital program [5].

Considering the four steps of the arrhythmia classification systems, this dataset can be seen as having finished the first three steps: preprocessing, segmentation and feature extraction, and is ready for the fourth step classification. It is believed that the raw ECG data, such as that in BIH-MIT arrhythmia dataset, can be converted to this UCI data frame format with each record corresponding to one person, and our algorithm can identify the arrhythmia type for each person.

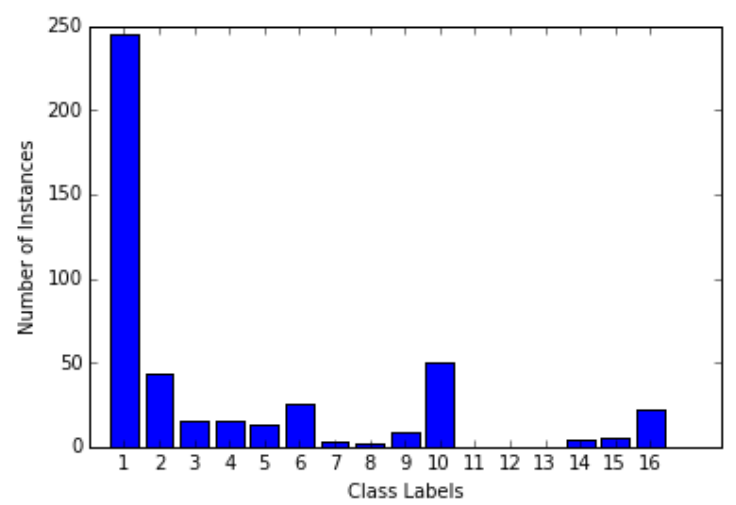

Fig. 1 Class Distribution in UCI Arrhythmia Dataset

\subsection{RELATED WORK}

Among dozens of learning algorithms in ECG classification literature, there are four most popular algorithms: support vector machines (SVM) [1], artificial neural networks (ANN) [8], linear discriminant (LD) [2], and Reservoir Computing with Logistic Regression (RC) [1]. SVM is efficient in both execution time, both in the training and in the testing, but it behaves badly for imbalanced classes. [8] stated that Multilayer Perceptrons (MLP) ANN was significantly superior than LD. [3] stated that RC was the state-of-the-art method for ECG classification.

There are no explicit logical relationships among these methods which can prove that one method is superior than another. It is the experiment result that can be used to compare their performances. In this work, the ensemble methods are explored, instead of the aforementioned methods.

\section{METHODOLOGY}

In this section, the overall design of the program is introduced, following by the core of the methodology which is machine learning models.

\subsection{PROGRAM STRUCTURE}

The program is designed as a pipeline: missing value processing, normalization, splitting the dataset into training set and test set, training, prediction, cross validation, and hyperparameter tuning. It is illustrated in Fig. 2. 


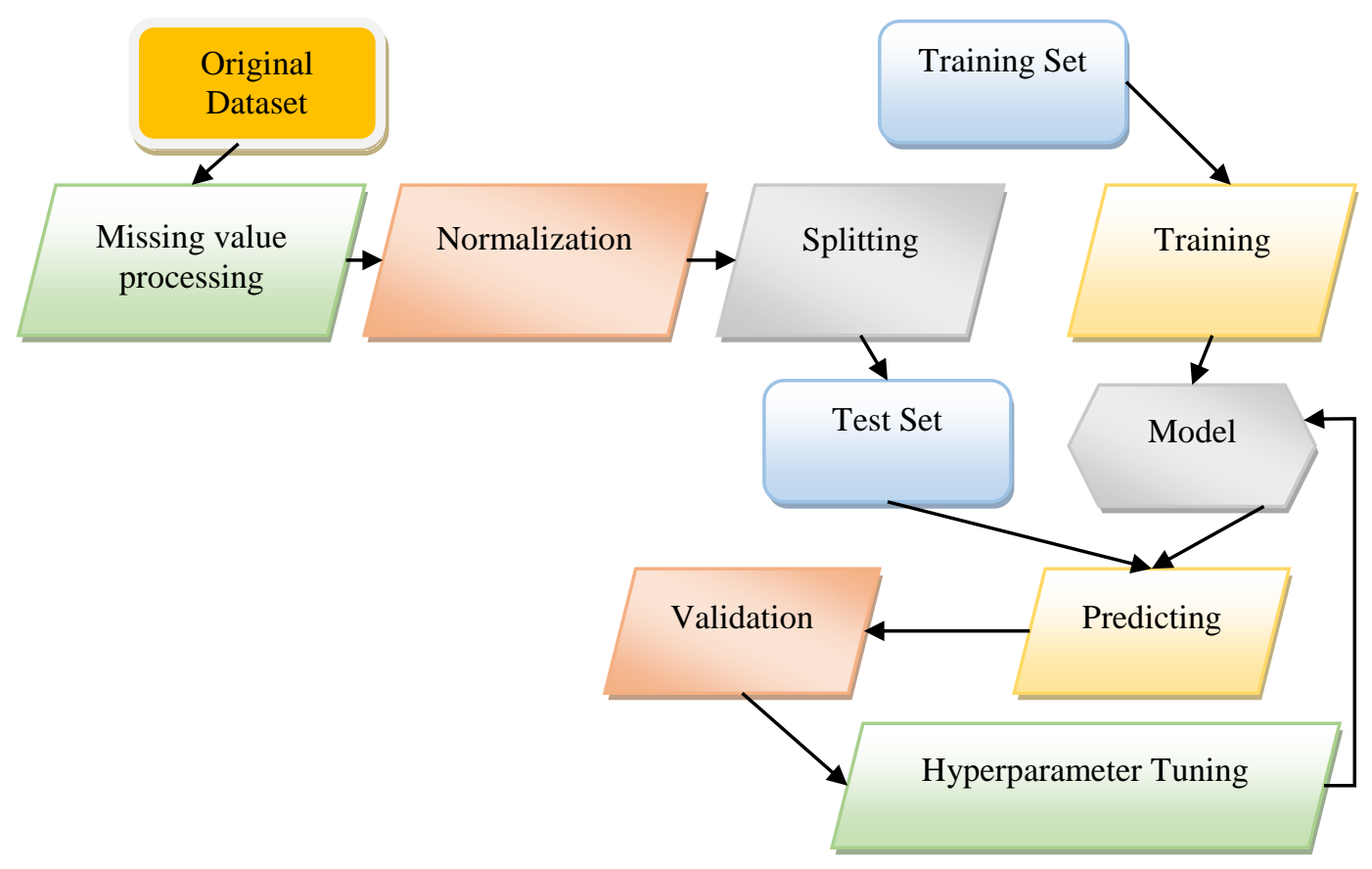

Fig. 2 Program Structure

This is a typical structure of a machine learning program. For the details, see section 4 .

The remaining part of this section introduces the models, including their training approaches.

\subsection{ENSEMBLE METHOD}

Ensemble method (meta-algorithm) combines more than one weak learners to create a strong learner. It is based on the question posed by Kearns and Valiant (1988, 1989): Can a set of weak learners create a single strong learner? In supervised learning, a weak learner is defined to be a classifier which is slightly correlated to the true classification (it can label examples better than random guessing). In contrast, a strong learner is a classifier that is arbitrarily well-correlated with the true classification.

There are two major types of ensemble methods. One is boosting which "boosts" the model to fit the training set by iteratively and addictively learning. The typical boosting method is Gradient Boosting which is the core of the library XGBoost. The other type is averaging which "averages" the prediction results of the component classifiers by a linear way of combination. One special case of the averaging methods is the bagging methods. Bagging means Bootstrap Aggregating. It ensembles the classifiers by combining classifications of randomly generated training sets. A typical bagging method is Random Forest. In this work, XGBoost and Random Forest are used. In addition, an averaging method votingClassifier is used to combine the three classifiers XGBoost, Random Forest and Logistic Regression.

\subsection{XGBOOST: GRADIENT BOOSTING}

XGBoost is an open-source library which provides the gradient boosting framework. From the project description, it aims to provide a "Scalable, Portable and Distributed Gradient Boosting (GBM, GBRT, GBDT) Library". It is used by many winning teams of a number of machine learning competitions.

XGBoost is short for "Extreme Gradient Boosting", where the term "Gradient Boosting" is proposed in paper [4]. XGBoost is based on this original model. 
Boosting is a kind of machine learning ensemble meta-algorithm (see section 4.1) for reducing bias and variance in supervised learning, and a family of machine learning algorithms which convert weak learners to strong ones [11]. Most boosting algorithms consist of iteratively learning weak classifiers with respect to a distribution and adding them to a final strong classifier. Algorithms that achieve hypothesis boosting quickly became simply known as "boosting". XGBoost is boosted regression trees, and the hyperparameters are learned in gradient boosting method.

A regression tree is a decision tree with each leaf containing one score. XGBoost ensembles multiple trees to decrease the classification variability. When XGBoost predicts the class for a record (a feature vector), it sums the scores for that class (corresponding to a leaf) predicted by each of the tree.

$$
\hat{y}_{i}=h_{\theta}\left(x_{i}\right)=\sum_{k=1}^{K} f_{k}\left(x_{i}\right), \quad f_{k} \in \mathcal{F}
$$

$f_{k}$ is a regression tree and is a function mapping attributes (features) to the score, and $\mathcal{F}$ is the space of all regression trees.

The parameters that need to be learned are the functions $f_{k}$. We use ${ }^{\theta}$ to denote the parameters:

$$
\Theta=\left\{f_{1}, f_{2}, \ldots, f_{K}\right\}
$$

The objective function is:

$$
I(\Theta)=\sum_{i=1}^{n} l\left(y_{i}, \hat{y}_{i}\right)+\sum_{k} \Omega\left(f_{k}\right), \quad f_{k} \in \mathcal{F}
$$

The gradient boosting method learns ${ }^{\theta}$ by addictive training. It starts from a constant prediction, and add a new function $f_{k}$ in each round. After $K$ rounds, ${ }^{\ominus}$ is completely constructed. In each round, $f_{k}$ is chosen by minimizing the corresponding $I(\theta)$. In the minimization computation, gradients of the loss function are used, so this method is called "gradient boosting". Detailed algorithm is discussed in [4].

\subsection{RANDOM FOREST}

Random Forest is essentially bootstrap aggregating. It is an ensemble method. A set of Decision Trees are created, on different datasets obtained by sampling with replacement. It utilizes feature bagging (not all features used, rather a random subset of features used for training of each decision tree).

In addition, when splitting a node during the construction of the tree, the split that is chosen is the best split among a random subset of the features. Thus, the bias of the forest usually slightly increases, but due to averaging its variance also decreases, usually more than compensating for the increase in bias, hence yielding an overall better model [10].

\subsection{LOGISTIC REGRESSION}

Logistic Regression is a linear model for classification. If sample $x_{i}$ has $\mathrm{n}$ features $\left(x_{i}^{(1)}, x_{i}^{(2)}, \ldots, x_{i}^{(n)}\right)$, then the hypothesis function of Logistic Regression is:

$$
h_{\theta}\left(x_{i}\right)=g\left(\theta^{T} x_{i}\right)=g\left(\theta_{0}+\theta_{1} x_{i}^{(1)}+\theta_{2} x_{i}^{(2)}+\cdots+\theta_{n} x_{i}^{(n)}\right)=\frac{1}{1+e^{-\theta^{T} x_{i}}}
$$


The above function $g\left(\theta^{T} x_{i}\right)$ is called logit function or sigmoid function. If $\theta^{T} x_{i} \geq 0$, then $g\left(\theta^{T} x_{i}\right) \geq 0.5$, we define $\hat{y}_{i}=1$, and if $\theta^{T} x_{i}<0$, then $g\left(\theta^{T} x_{i}\right)<0.5$, we define $\hat{y}_{i}=0$

This hypothesis function defines a binary classifier. In multiclass case, as in this ECG classification problem, a set of logistic regression classifiers must be created, with each classifier for one class. This strategy is called one-vs-rest scheme (OvR) Error! Reference source not found.. scikit-learn LogisticRegression uses OvR by default.

The objective function for Logistic Regression is discussed in section 4.7.1.

\section{IMPLEMENTATION AND ANALYTICS}

In the UCI Arrhythmia Dataset, the data file is "arrhythmia.data" which is the same as a CVS file. In this file, the missing data is denoted as "?". The file "arrhythmia.names" is the codebook for this dataset. Python packages scikit-learn, numpy, pandas and matplotlib.pyplot are used to do the experiments.

First, the missing value symbols "?" are replaced with empty strings "", and the new data is saved into a new file. Then pandas.read_csv( ) is called to read the whole dataset as a pandas DataFrame. Then the first 279 columns are extracted as variable $\mathrm{X}$ which is the 279 features. The $280^{\text {th }}$ column is stored in variable y which is the labels for the 452 records. After the above preparation, we impute the missing values, splitting the dataset into a training set and a test set, then we train XGBoost, RandomForest and LogisticRegression models and use them to classify the test set. By 10-fold cross validation, we get the average performance of the models on this dataset. We devise an ensemble voting method to combine the previous 3 models to reduce the variance of the classification, and get a result that is better than any of the single models.

\subsection{PREPROCESSING: MISSING DATA PROCESSING AND NORMALIZATION}

The empty strings between the delimiter "," in the CSV file is read and interpret as NaN in pandas. NaN can be replaced by meaningful values by sklearn. preprocessing. Imputer.

$X=$ Imputer(missing_values $=$ 'NaN', strategy='mean', axis $=0)$.fit_transform $(X)$

missing_values='NaN' means all the values encoded as np.nan will be imputed. strategy='mean' means replacing the missing values using the mean along the axis. axis $=\theta$ means imputing along columns. The above line of code iterates each column and impute its missing values by the mean value of that column.

\subsection{PREPROCESSING: NORMALIZATION}

sklearn.preprocessing.StandardScaler is used to normalize the data X.

$X=$ StandardScaler (). fit_transform $(X)$

StandardScaler standardizes features by removing the mean and scaling to unit variance.

\subsection{SPLITTING DATASET INTO TRAINING SET AND TEST SET}

sklearn.model_selection.train_test_split() function is used to shuffle the records in $X$ and to partition them into a training set and a test set. The code is as follows: 


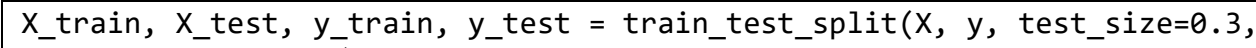

This line of code randomly selects $70 \%$ of records in $x$ and $y$, and group them in the training set, the remaining records are grouped in the test set.

random_state $=1$ is a seed to the pseudo-random number generator, passing 1 as random_state value makes sure the pseudo-random series generated is the same in multiple calls of this function.

\subsection{TRAINING AND PREDICTION}

In scikit-learn, the Python code for training and prediction follows the same pattern:

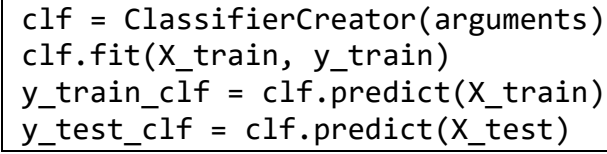

The three models used in this work are created by the following code:

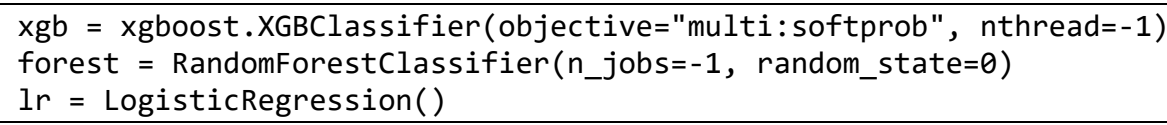

\subsection{VALIDATION}

The simplest validation is to compare the prediction result and the label. Comparing y_train_clf and y_train, we can get the accuracy rate the fitted model can get on the training set. Comparing y_test_clf and y_test, we can get the accuracy the model can predict on the test set. np.mean(y_test_clf $==y_{-}$test) gives the accurate in percentage. The results are in Table and Table.

We can also use the following code to calculate the accuracy. The result is the same as the above method.

clf.score(X_train, y_train)

clf.score(X_test, y_test)

4.5.1 10-FOLD CROSS VALIDATION: Compared to the above validation method, 10 -fold cross validation is in general an applicable and better way to predict the performance of a model.

In 10 -fold cross validation, the original sample is randomly partitioned into 10 equal sized subsamples. The fitting and prediction process is run 10 times on 10 different partitions for training set and test set. Each iteration runs on a partition which choose one subsample as the test set, and the remaining subsamples as the training set. Thus the 10fold running can get 10 test scores. The mean of these scores is the average accuracy for the model.

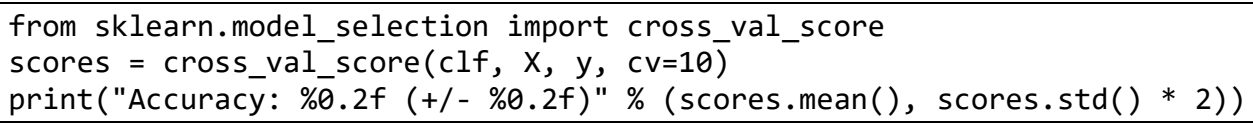

The validation results are in Table and Table . 


\subsection{ENSEMBLE VOTING METHOD}

The idea of ensemble voting method is to combine different classifiers by majority vote (hard vote) or the average predicted probabilities (soft vote) to predict the class labels. It can balance out the individual weaknesses of the classifiers.

Hard vote simply takes the majority predicted label as the result. Soft vote is more complicated and usually performs better than hard vote. In this work, soft vote is used to combine the classifiers mentioned above.

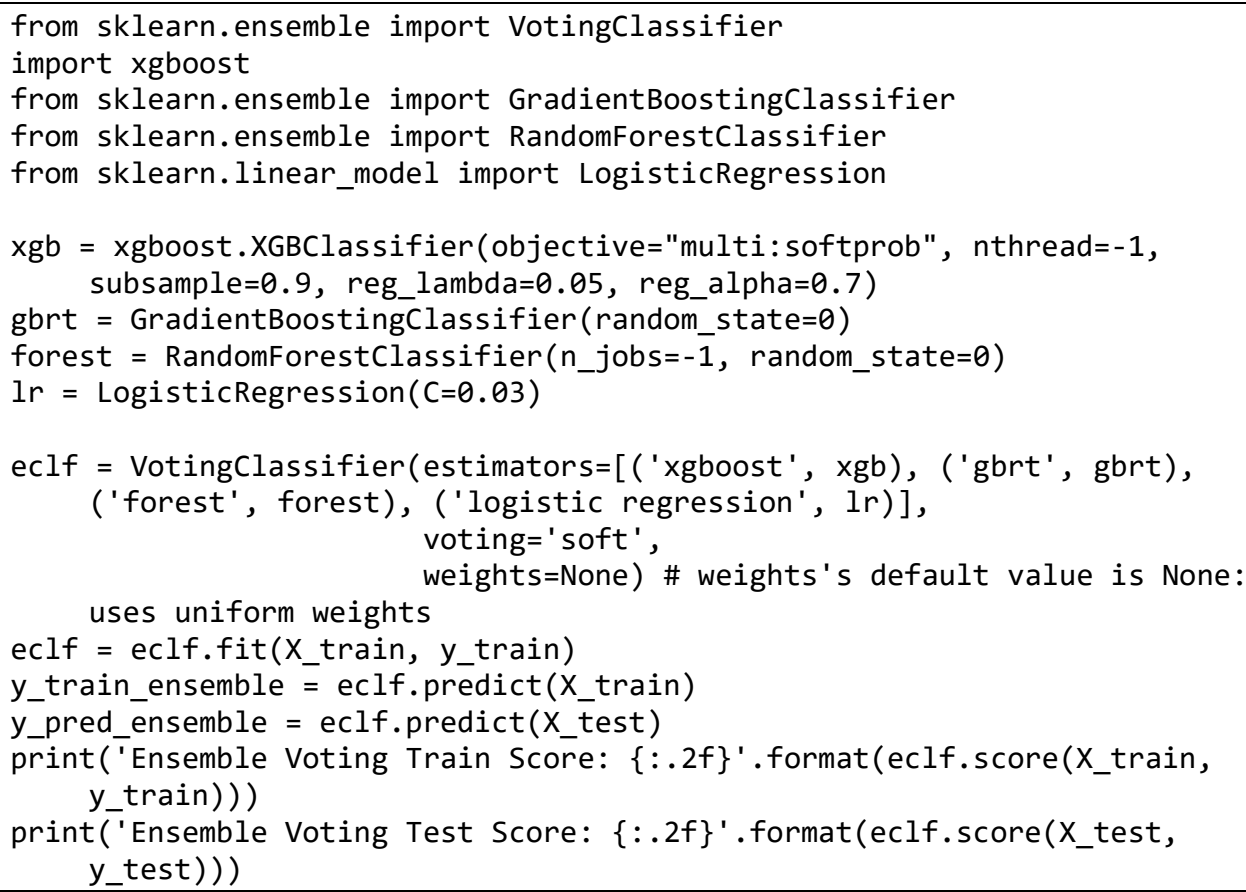

The results in Table and Table show that ensemble voting classifier achieves the best prediction accuracy. Because it balances out the weaknesses of the classifiers combined.

\subsection{GRID SEARCH AND HYPERPARAMETER TUNING}

Table shows that the training accuracies are very high, and the test accuracies are relatively low, which is a sign of overfitting. Overfitting, or high variance, is caused by a hypothesis function that fits the available data but does not generalize well to predict new data. It is usually caused by a complicated function that creates a lot of unnecessary curves and angles unrelated to the data. There are three main options to address the issue of overfitting: (1) reduce the number of features; (2) regularization; (3) tune classifier hyperparameters. Hyperparameters are parameters that are not directly learnt within estimators. The regularization parameter $\mathrm{C}$ is a typical example. In XGBoost, there are 15 hyperparameters that are passed to the constructor, including subsample, reg_lambda, reg_alpha, etc.

Grid Search exhaustively searches through a manually specified subset of the hyperparameter space of a learning algorithm, then compares the cross-validation performances of different configurations of these hyperparameters, and chooses the best configuration. We applied regularization on logistic regression, and we applied grid search on the XGBoost to get the best hyperparameters for it.

4.7.1. REGULARIZATION ON LOGISTIC REGRESSION: In LogisticRegression classifier, there is a parameter $\mathrm{C}$ that controls the regularization. Parameter $\mathrm{C}$ must be a positive float. Smaller values specify stronger regularization. By default, $\mathrm{C}=1.0$ which 
means no regularization. We use the default penalty (regularization type) 'L2'. The cost function with 'L2' penalty for scikit-learn LogisticRegression is:

$$
I(\theta)=\Omega(\theta)+\mathrm{L}(\theta)=\frac{1}{2} \theta^{T} \theta+C \sum_{i=1}^{n} \log \left(\exp \left(-y_{i}\left(X_{i}^{T} \theta+c\right)\right)+1\right)
$$

The magnitude of the weight $\theta$ is restricted by smaller c. In the experiment, we got the following result, see TableI and Fig. 3 .

Table I. Regularization Parameter C and Estimator Performance

\begin{tabular}{|l|l|l|l|l|l|l|l|l|l|l|}
\hline C & 1 & 0.8 & 0.5 & 0.1 & 0.08 & 0.07 & $\mathbf{0 . 0 5}$ & $\mathbf{0 . 0 4}$ & $\mathbf{0 . 0 3}$ & $\mathbf{0 . 0 1}$ \\
\hline $\begin{array}{l}\text { Training } \\
\text { accuracy }\end{array}$ & 0.99 & 0.99 & 0.97 & 0.92 & 0.9 & 0.9 & $\mathbf{0 . 8 9}$ & 0.88 & 0.87 & 0.83 \\
\hline Test accuracy & 0.71 & 0.71 & 0.73 & 0.77 & 0.78 & 0.78 & $\mathbf{0 . 7 9}$ & 0.79 & 0.77 & 0.77 \\
\hline
\end{tabular}

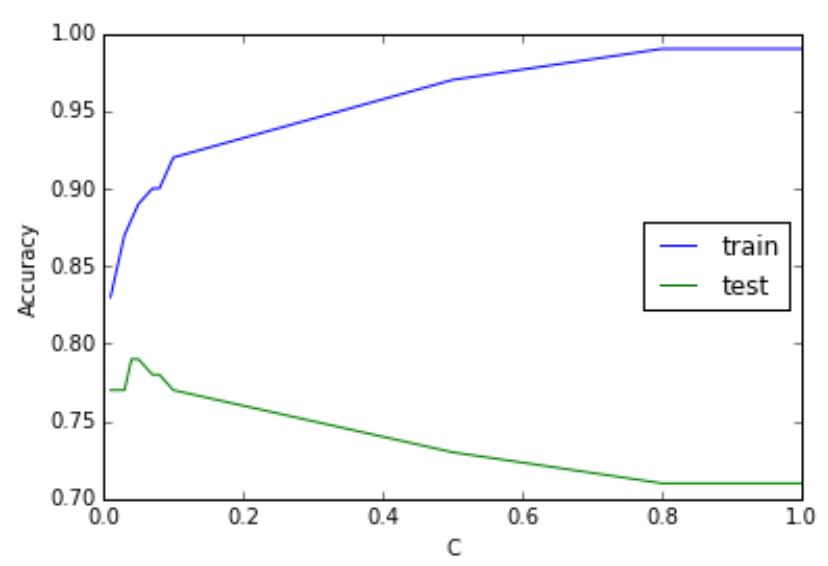

Fig. 3 Regularization Parameter C and Estimator Performance

Table 1 shows that the best performance is achieved when $\mathrm{C}=0.05$.

By Grid Search, we find that $\mathrm{C}=0.03$ gets the best performance. This is because GridSearchCV uses 3-fold cross-validation which is different from and better than the above 1 -fold validation. We believe $\mathrm{C}=0.03$ is the best value.

Selecting $C=1.0,0.1$, and 0.03 sequentially, the learning curve is drawn as Fig. 4. The blue lines are for $\mathrm{C}=1.0$, the red lines are for $\mathrm{C}=0.1$, the purple lines are for $\mathrm{C}=0.03$. This figure shows that $\mathrm{C}=0.03$ performs the best. 


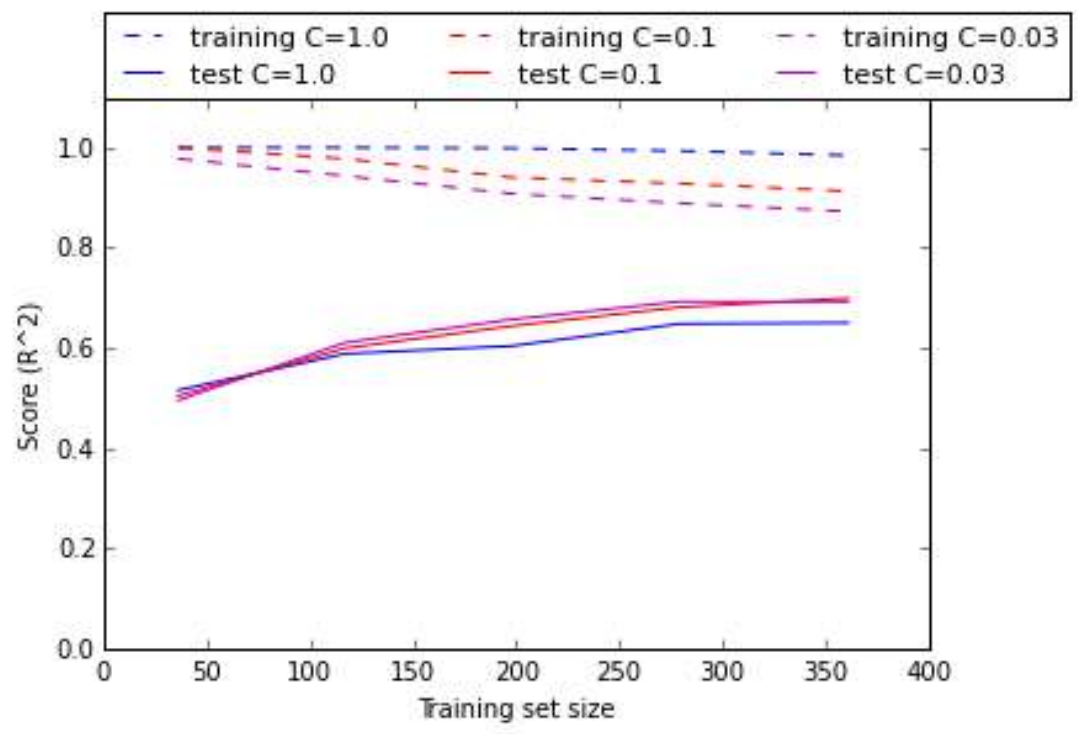

Fig. 4 Learning Curves for LogisticRegression with $C=1.0,0.1,0.03$

4.7.2. GRID SEARCH ON XGBOOST: In XGBoost, we tune these parameters: subsample, reg_lambda, reg_alpha. subsample is the ratio of the training instance. reg_lambda is the L2 regularization term on weights. reg_alpha is the L1 regularization term on weights.

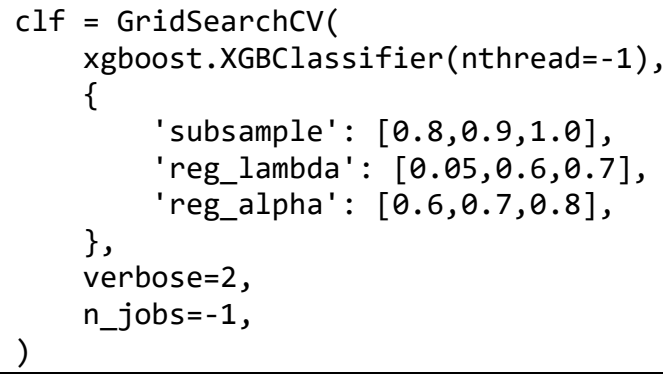

The above GridSearchCV performs 3-fold cross-validation by default, and uses score method as the scoring function by default.

\{'reg_alpha': 0.7, 'reg_lambda': 0.05, 'subsample': 0.9$\}$ is returned by GridSearchCV's best_params_attribute.

By clf.score(X_train, y_train) and clf.score(X_test, y_test), we get the best training score and test score as $\mathbf{1 . 0}$ and $\mathbf{0 . 7 6}$ respectively.

\subsection{PLOT LEARNING CURVES}

A learning curve is a graphical representation of the increase of learning with experience. Ideally, with the increase of the training samples, the test prediction accuracy should get close to the training prediction accuracy. Following is the code for plotting the learning curves for all the tuned classifiers.

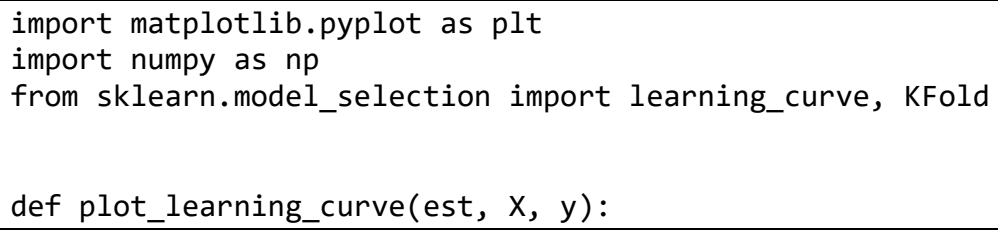




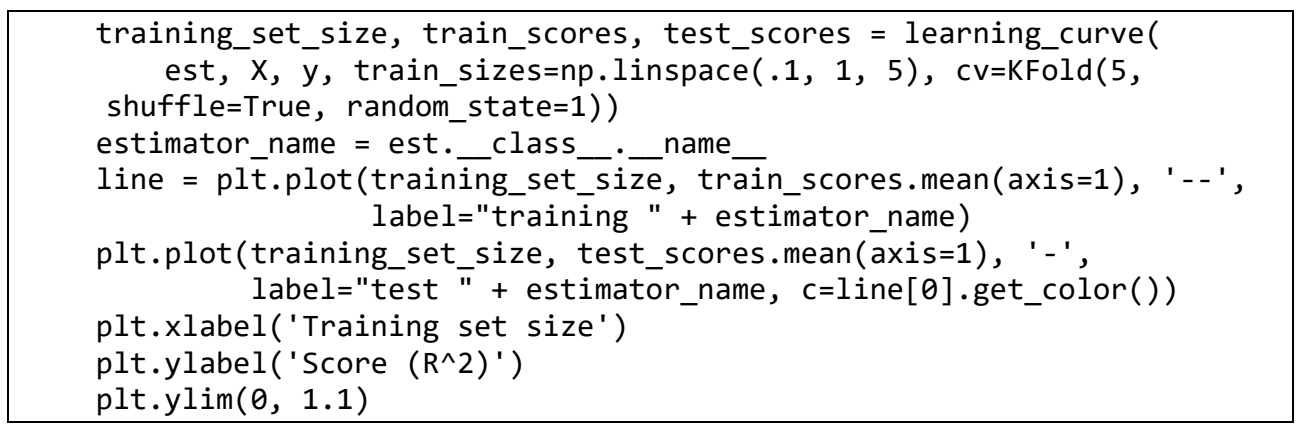

\subsection{PLOT CONFUSION MATRIX}

A confusion matrix, also known as an error matrix, is a specific table layout that allows visualization of the performance of an algorithm, typically a supervised learning one. Each column of the matrix represents the instances in a predicted class while each row represents the instances in an actual class. Following is the code for plotting the learning curves for all the tuned classifiers. Fig. 5 to Fig. 8 are confusion matrices.

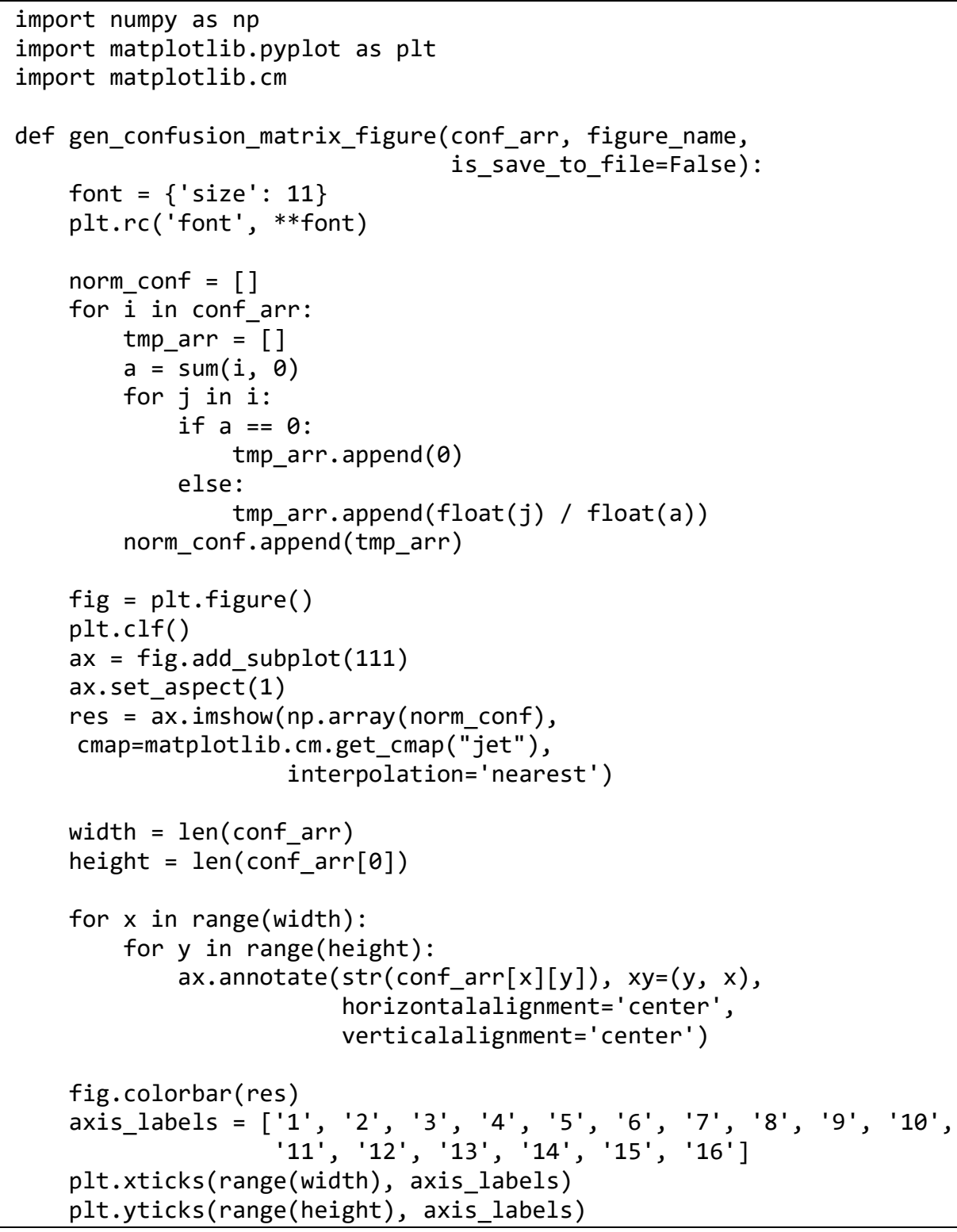




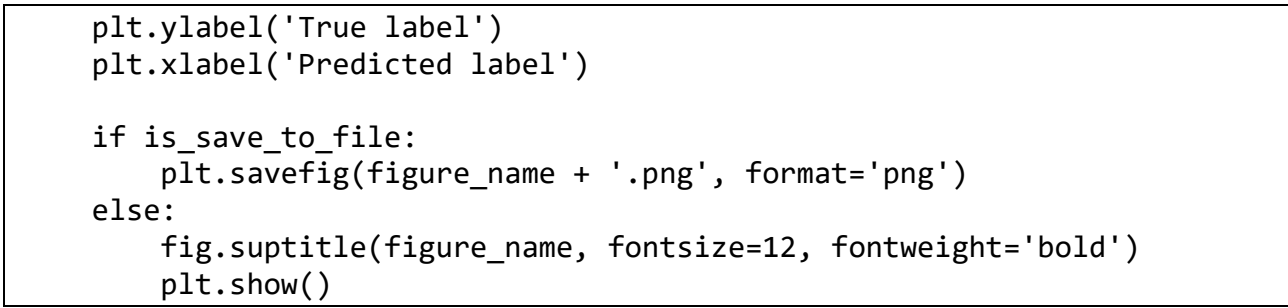

\subsection{RESULTS}

For Classification Report which includes precision, recall, f1-score, and number of support samples, refer to the appendix. Following are tables and figures for the basic measurements of the classification performance.

Table II. 1-fold Validation for Default Classifiers

\begin{tabular}{|l|l|l|l|l|}
\hline & XGBoost & RandomForest & LogisticRegression & $\begin{array}{l}\text { EnsembleVoting } \\
\text { One }\end{array}$ \\
\hline $\begin{array}{l}\text { Training } \\
\text { accuracy }\end{array}$ & 1.00 & 0.98 & 0.99 & 1.00 \\
\hline $\begin{array}{l}\text { Test } \\
\text { accuracy }\end{array}$ & 0.75 & 0.71 & 0.71 & $\mathbf{0 . 7 8}$ \\
\hline
\end{tabular}

The Ensemble VotingClassifier used in this table is built upon the default XGBoost, RandomForest and LogisticRegression.

Table III. 1-fold validation for Default Classifiers and Tuned Classifiers

\begin{tabular}{|l|l|l|l|l|}
\hline & $\begin{array}{l}\text { XGBoost } \\
\text { default }\end{array}$ & $\begin{array}{l}\text { XGBoost } \\
\text { tuned }\end{array}$ & $\begin{array}{l}\text { LogisticRegression } \\
\text { default }\end{array}$ & $\begin{array}{l}\text { LogisticRegression } \\
\text { tuned }\end{array}$ \\
\hline $\begin{array}{l}\text { Training } \\
\text { accuracy }\end{array}$ & 1.00 & 1.00 & 0.99 & 0.89 \\
\hline $\begin{array}{l}\text { Test } \\
\text { accuracy }\end{array}$ & 0.75 & 0.77 & 0.71 & $\mathbf{0 . 7 9}$ \\
\hline
\end{tabular}

XGBoost and LogisticRegression used in this table are tuned by Grid Search. \{'C': 0.03$\}$ for

LogisticRegression. \{'reg_alpha': 0.7, 'reg_lambda': 0.05, 'subsample': 0.9\} for XGBoost.

Table IV. 10-fold Validation for Default Classifiers

\begin{tabular}{|l|l|l|l|l|}
\hline & XGBoost & RandomForest & LogisticRegression & $\begin{array}{l}\text { EnsembleVoting } \\
\text { One }\end{array}$ \\
\hline $\begin{array}{l}\text { Test } \\
\text { accuracy }\end{array}$ & $0.74 \pm 0.10$ & $0.72 \pm 0.10$ & $0.72 \pm 0.13$ & $\mathbf{0 . 7 5} \pm \mathbf{0 . 1 5}$ \\
\hline
\end{tabular}

XGBoost and LogisticRegression used in this table are default without tuning.

Table V. 10-fold Validation for Tuned Classifiers

\begin{tabular}{|l|l|l|l|l|}
\hline & $\begin{array}{l}\text { XGBoost } \\
\text { tuned }\end{array}$ & RandomForest & $\begin{array}{l}\text { LogisticRegression } \\
\text { tuned }\end{array}$ & $\begin{array}{l}\text { EnsembleVoting } \\
\text { Two }\end{array}$ \\
\hline $\begin{array}{l}\text { Test } \\
\text { accuracy }\end{array}$ & $0.75 \pm 0.11$ & $0.72 \pm 0.10$ & $0.72 \pm 0.13$ & $\mathbf{0 . 7 6} \pm \mathbf{0 . 1 3}$ \\
\hline
\end{tabular}

XGBoost and LogisticRegression used in this table are tuned by Grid Search. \{'C': 0.03$\}$ for

LogisticRegression. \{'reg_alpha': 0.7, 'reg_lambda': 0.05, 'subsample': 0.9\} for XGBoost. 


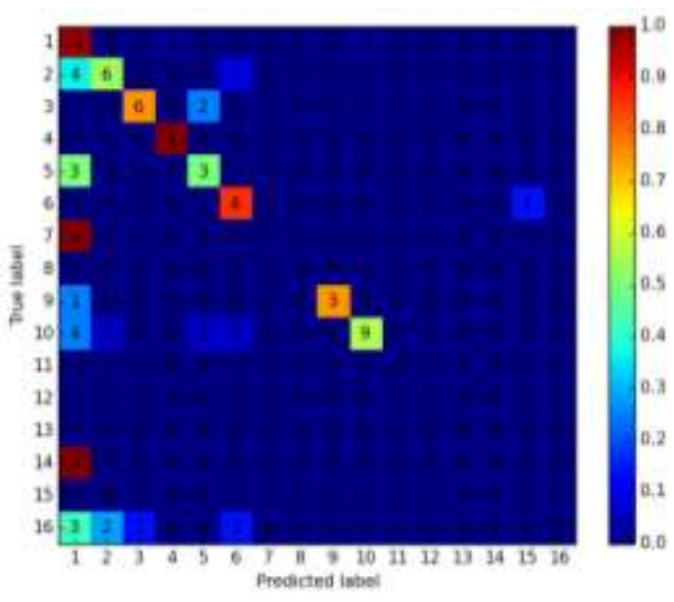

XGBoost Test

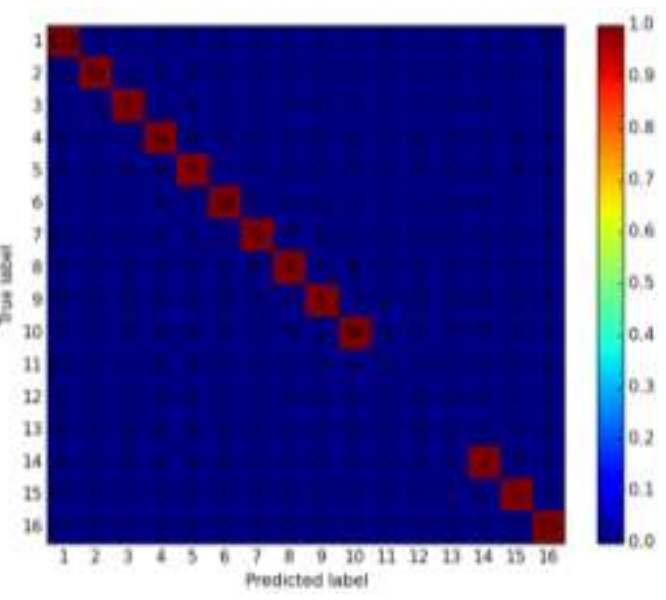

XGBoost Train

Fig. 5 Confusion Matrix for XGBoost

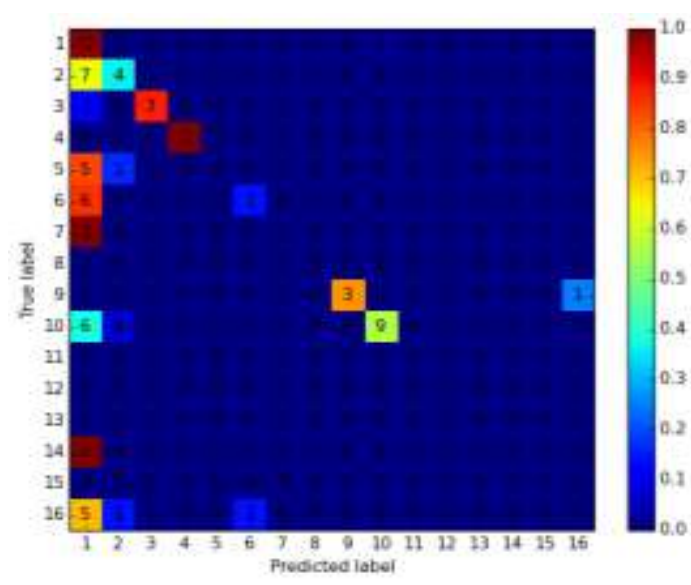

RandomForest Test

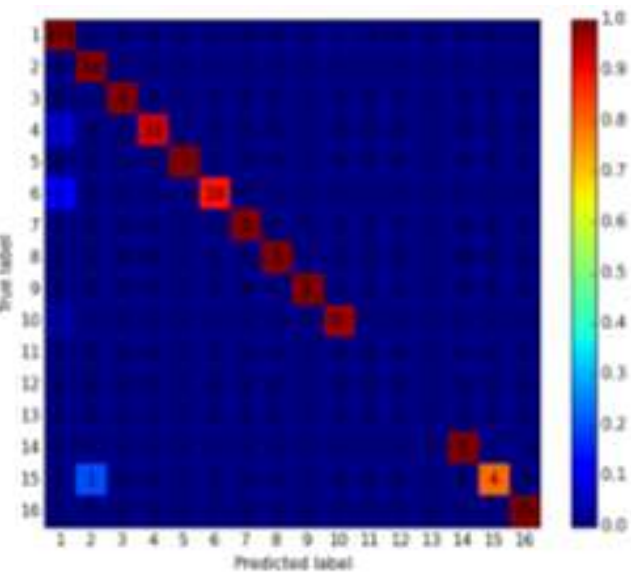

RandomForest Train

Fig. 6 Confusion Matrix for RandomForest

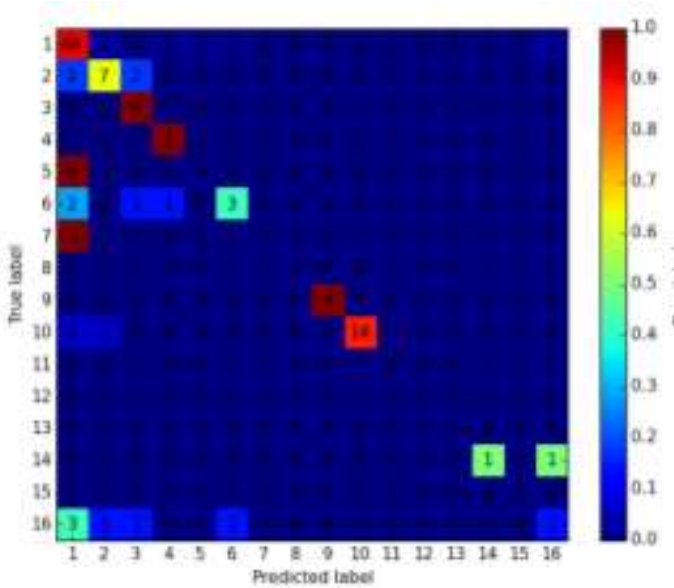

LogisticRegression Test

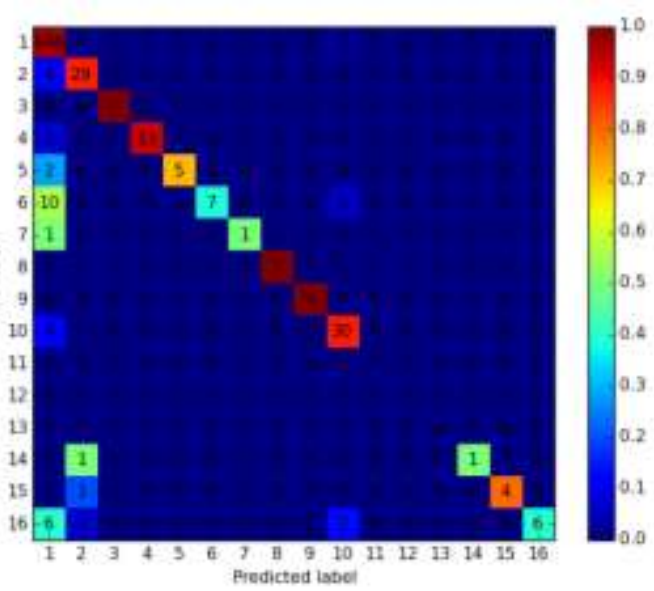

LogisticRegression Train

Fig. 7 Confusion Matrix for LogisticRegression 

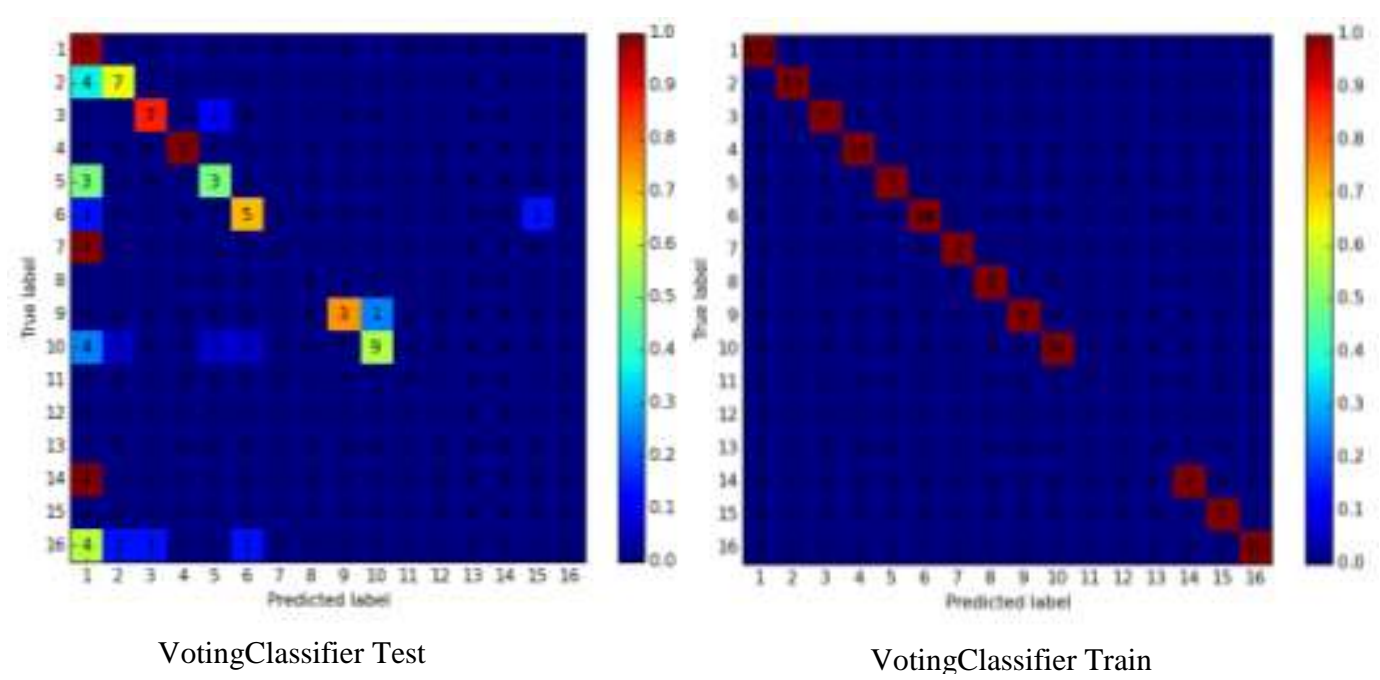

Fig. 8 Confusion Matrix for VotingClassifier

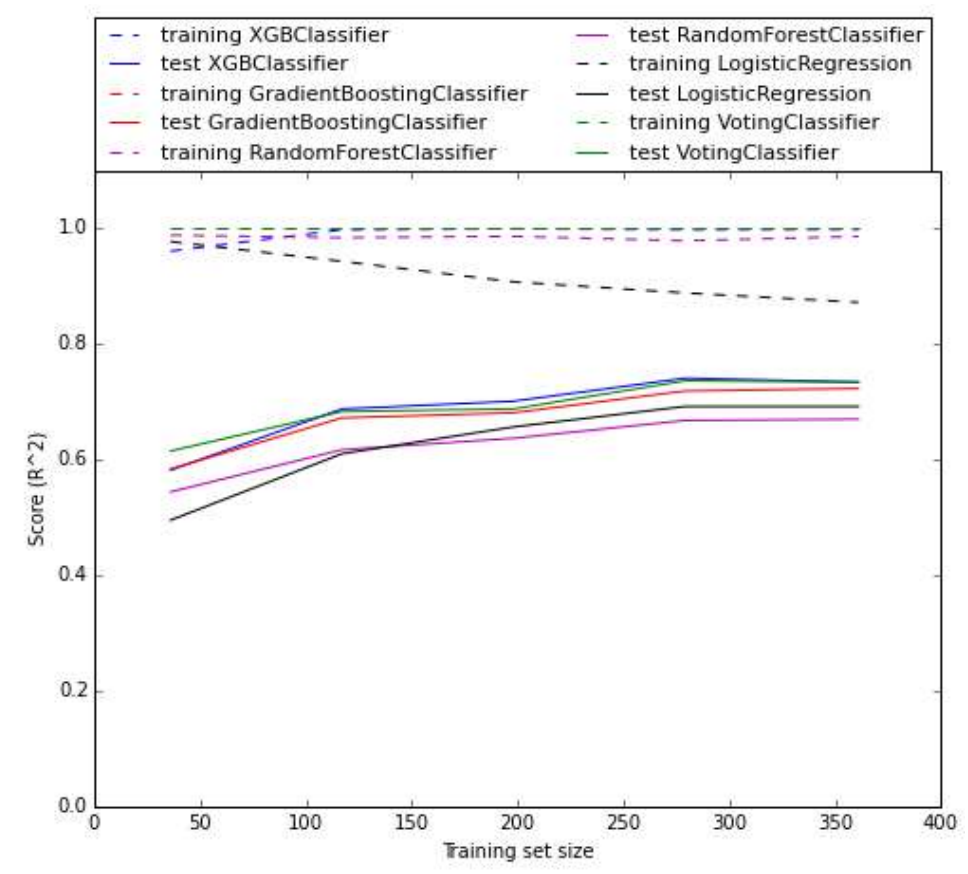

Fig. 9 Learning Curves for All Tuned Classifiers

\subsection{DISCUSSION}

Table 2 and 3 are the results of 1-fold validation. They compare the default classifiers, tuned XGBoost and tuned LogisticRegression. LogisticRegression performs the best, and is slightly better than ensemble VotingClassifier. Ensemble VotingClassifier outperforms all the other classifiers, which is reasonable according to the theory of the ensemble method. Table 4 and 5 are the results of 10 -fold cross-validation which is a better metrics for the classifiers' performances. In these results, VotingClassifier outperforms all the other ones in terms of the mean accuracy.

From the above tables, we can see that although LogisticRegression with regularization $C=0.03$ performs better than its $C=1.0$ counterpart in the 1 -fold validation, it does the same as the $C=1.0$ one in the 10 -fold validation, which means that the improvement is trivial. 10-fold validation is believed to reflect the true performance better than 1-fold validation on the real sophisticated data. Fig. 9 shows that as the training size 
increases, the test score (accuracy) increases. The green line for Votingclassifier indicates the best test score.

In all, the ensemble Votingclassifier is the winner in our work for this UCI Arrhythmia Dataset. Comparing with HA Guvenir's result 68\% accuracy [5], all our classifiers are better than HA Guvenir's VFI5 classifier. Albert gets $75.7 \%$ for Neural Network and $76.0 \%$ for Random Forest [6]. In our work, in 1-fold validation, our ensemble votingClassifier achieves $78 \%$, and in 10-fold validation, our ensemble VotingClassifier achieves $76 \%$ which is at least as good as Albert's Random Forest. Albert used the same dataset, but in paper [6] he does not tell us which validation method he used.

\section{CONCLUSIONS}

This work takes a model selection approach to select, explore and design classifiers. We applied some classic classifiers on all the 279 features of the UCI Arrhythmia Dataset, and predict the heartbeat categories for the records. Each record corresponds to a person, and each record has only one label for its category. Two scikit-learn built-in ensemble classifiers XGBoost and RandomForest perform better than even well-tuned Logistic- Regression. We build VotingClassifier based on the ensemble voting metaalgorithm and three built-in classifiers, and get a stronger classifier that outperforms even well-tuned XGBoost and RandomForest. The best multiclass classification accuracy $78 \%$ is achieved by our votingClassifier. This work shows that, as a meta-algorithm, the ensemble method can guide the design of strong classifiers based on a set of weak classifiers.

There are a lot of meaningful future work for full-automatic ECG arrhythmia classification based on the current achievement. First, to further reduce variance, reducing features may help. Feature selection can be used, such as Principle Component Analysis (PCA) or Independent Component Analysis (ICA). Another method is to manually select some features. Second, there is a large unbalance in the dataset, see Fig. 1. Majority of the records are of the normal type. Two categories are empty. The imbalance can make the performance bad for some classifiers, such as Support Vector Machine [7]. The consequences of the data unbalance for our classifiers need to be analyzed. Third, more measurements can be considered for the evaluation of the performance. The authors in [7] introduces AAMI's (the Association for the Advancement of Medical Instrumentation) recommendation: Overall accuracy $(A c c)$, Sensitivity $(S e)$, and Positive predictivity $(+P)$. In this work, only $A c c$ is considered. $S e$ and $+P$ should be added in the future work. Fourth, more kinds of classifiers can be tried and compared on this dataset, such as AdaBoost, Reservoir Computing with Logistic Regression (RC) [1]. Fifth, these classifiers and model selection and hyperparameter tuning method can be applied to MITBIH Arrhythmia Dataset [9] and other ECG datasets for per-heartbeat classification, which is closer to the applications in the real world. Sixth, the training time and prediction time should be evaluated. Since real-time classification is an important application in the real world, and it requires fast prediction time. Lastly, based on the research in this work, a complete ECG arrhythmia full automatic identification system can be built. The system consists of the 4 major steps (components) mentioned in the introduction section.

\section{Appendix: Classification Report}

The classification report which includes the measurements precision, recall, f1-score and support can be produced by the following code. The report output is listed after the code. 


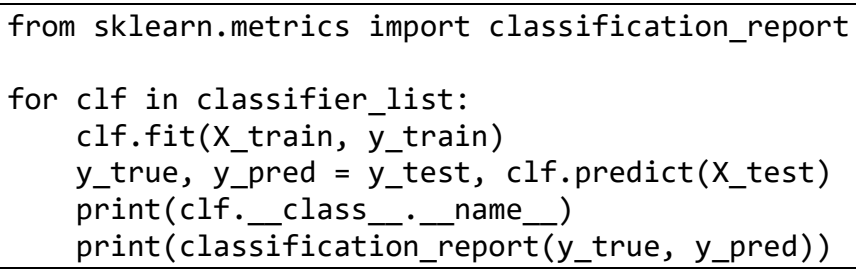

XGBClassifier

$$
\text { precision }
$$

recall

f1-score

support

$$
\begin{aligned}
& 0.80 \\
& 0.67 \\
& 0.86 \\
& 0.50 \\
& 0.50 \\
& 0.67 \\
& 0.00 \\
& 1.00 \\
& 0.90 \\
& 0.00 \\
& 0.00 \\
& 0.00
\end{aligned}
$$

$$
0.97
$$$$
0.88
$$

0.55

0.75

1.00

0.50

0.86

0.00

0.75

0.56

0.00

0.00

0.00

0.60

0.80

0.67

0.50

0.75

0.00

0.86

0.69

0.00

0.00

0.00

$\operatorname{avg} /$ total

0.73

0.77

0.74

136

GradientBoostingClassifier

$$
\text { precision }
$$

recall

f1-score

support

$$
\begin{aligned}
& 0.80 \\
& 0.64 \\
& 0.75 \\
& 0.50 \\
& 0.75 \\
& 0.83 \\
& 0.00 \\
& 1.00 \\
& 0.83 \\
& 0.00 \\
& 0.00 \\
& 0.00
\end{aligned}
$$

0.96

0.64

0.75

1.00

0.50

0.71

0.00

0.75

0.62

0.00

0.00

0.00

0.87

0.64

0.75

0.67

0.60

0.77

0.00

0.86

0.71

0.00

0.00

0.00

$\operatorname{avg~/~total~}$

0.73

0.77

0.74

136

RandomForestClassifier precision

recall

f1-score

support

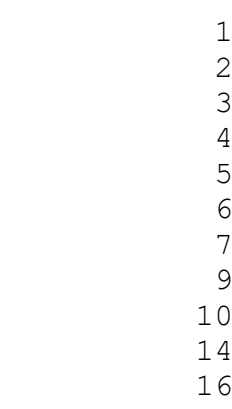

$$
\begin{aligned}
& 0.69 \\
& 0.57 \\
& 1.00 \\
& 1.00 \\
& 0.00 \\
& 0.50 \\
& 0.00 \\
& 1.00 \\
& 0.90 \\
& 0.00 \\
& 0.00
\end{aligned}
$$

0.99

0.81

0.44

0.36

0.88

1.00

0.00

0.14

0.00

0.75

0.56

0.00

0.00

0.93

1.00

0.00

0.22

0.00

0.86

0.69

0.00

0.00

0.71

0.65

136

LogisticRegression

$$
\begin{array}{rrrrr} 
& \text { precision } & \text { recall } & \text { fl-score } & \text { support } \\
1 & 0.81 & 0.90 & 0.86 & 73
\end{array}
$$




$\begin{array}{rrrrr}2 & 0.64 & 0.64 & 0.64 & 11 \\ 3 & 0.67 & 1.00 & 0.80 & 8 \\ 4 & 0.33 & 1.00 & 0.50 & 1 \\ 5 & 0.00 & 0.00 & 0.00 & 6 \\ 6 & 0.60 & 0.43 & 0.50 & 7 \\ 7 & 0.00 & 0.00 & 0.00 & 1 \\ 9 & 1.00 & 1.00 & 1.00 & 4 \\ 10 & 1.00 & 0.88 & 0.93 & 16 \\ 14 & 1.00 & 0.50 & 0.67 & 2 \\ 16 & 0.25 & 0.14 & 0.18 & 7 \\ \text { avg / total } & 0.74 & 0.77 & 0.75 & 136 \\ \text { VotingClassifier } & & & & \\ & \text { precision } & \text { recal1 } & \text { f1-score } & \text { support } \\ 1 & 0.79 & 0.97 & 0.87 & 73 \\ 2 & 0.78 & 0.64 & 0.70 & 11 \\ 3 & 0.88 & 0.88 & 0.88 & 8 \\ 4 & 0.50 & 1.00 & 0.67 & 1 \\ 5 & 0.60 & 0.50 & 0.55 & 6 \\ 6 & 0.71 & 0.71 & 0.71 & 7 \\ 7 & 0.00 & 0.00 & 0.00 & 1 \\ 9 & 1.00 & 0.75 & 0.86 & 4 \\ 10 & 0.82 & 0.56 & 0.67 & 16 \\ 14 & 0.00 & 0.00 & 0.00 & 0 \\ 15 & 0.00 & 0.00 & 0.00 & 136 \\ 16 & 0.00 & 0.00 & 0.00 & \end{array}$

\section{REFERENCES}

[1] Bazi, Yakoub, Naif Alajlan, Haikel AlHichri, and Salim Malek. "Domain adaptation methods for ECG classification." 2013 international conference on computer medical applications (ICCMA). IEEE, 2013.

[2] De Chazal, Philip, Maria O'Dwyer, and Richard B. Reilly. "Automatic classification of heartbeats using ECG morphology and heartbeat interval features." IEEE Transactions on Biomedical Engineering 51, no. 7 (2004): 1196-1206.

[3] Escalona-Morán, Miguel Angel, Miguel C. Soriano, Ingo Fischer, and Claudio R. Mirasso. "Electrocardiogram classification using reservoir computing with logistic regression." IEEE journal of biomedical and health informatics 19, no. 3 (2015): 892-898.

[4] Friedman, Jerome H. "Greedy function approximation: a gradient boosting machine." Annals of statistics (2001): 1189-1232.

[5] Guvenir, H. Altay, Burak Acar, Gulsen Demiroz, and Ayhan Cekin. "A supervised machine learning algorithm for arrhythmia analysis." In Computers in Cardiology 1997, pp. 433-436. IEEE, 1997.

[6] Haque, Albert. "Cardiac Dysrhythmia Detection with GPU-Accelerated Neural Networks." (2015).

[7] Luz, Eduardo José da S., William Robson Schwartz, Guillermo Cámara-Chávez, and David Menotti. "ECG-based heartbeat classification for arrhythmia detection: A survey." Computer methods and programs in biomedicine 127 (2016): 144-164.

[8] Mar, Tanis, Sebastian Zaunseder, Juan Pablo Martínez, Mariano Llamedo, and Rüdiger Poll. "Optimization of ECG classification by means of feature selection." IEEE transactions on Biomedical Engineering 58, no. 8 (2011): 2168-2177.

[9] Moody, George B., and Roger G. Mark. "The impact of the MIT-BIH arrhythmia database." IEEE Engineering in Medicine and Biology Magazine 20, no. 3 (2001): 45-50.

[10] scikit-learn documentation version 0.18.1. "1.11. Ensemble methods". April 2017. http://scikit-learn.org/stable/modules/ensemble.html

[11] Wikipedia. "Boosting (machine learning)." April 2017. https://en.wikipedia.org/wiki/Boosting_\%28machine_learning\%29\#cite_note-Kearns88-3

[12] Wikipedia. "Multiclass classification." April 2017. https://en.wikipedia.org/wiki/Multiclass_classification 
International Journal of Control and Automation Vol. 12, No. 4 (2019) 